УДК 517.51

\author{
V. K. Maslyuchenko, O. V. Maslyuchenko, O. D. Myronyk
}

\title{
ON L-SEPARATEDNESS AND L-REGULARITY OF THE CEDER PRODUCTS
}

\begin{abstract}
V. K. Maslyuchenko, O. V. Maslyuchenko, O. D. Myronyk. On L-separatedness and Lregularity of the Ceder products, Mat. Stud. 43 (2015), 78-87.

We study properties of the Ceder product $X \times_{b} Y$ of topological spaces $X$ and $Y$ with distinguished point $b \in Y$, recently introduced by the authors. Important examples of the Ceder product are the Ceder plane, the Alexandroff double circle and the Alexandroff duplicate. In particular, we detect Ceder products which are $L$-separated or $L$-regular (these notions generalize the separation axioms $T_{i}$ for $\left.i \in\left\{1,2,2 \frac{1}{3}, 2 \frac{1}{2}, 3,3 \frac{1}{2}\right\}\right)$.

В. К. Маслюченко, О. В. Маслюченко, О. Д. Мироник. L-отделимость и L-регулярность произведений Сидра // Мат. Студії. - 2015. - Т.43, №1. - С.78-87.

Изучаются свойства введенного авторами понятия произведения Сидра $X \times_{b} Y$ топологических пространств $X$ и $Y$, где $b \in Y$. Примерами произведения Сидра служат плоскость Сидра, двойная окружность Александрова, или ее обобщение, удвоение по Александрову. В частности, изучаются условия, при которых произведение Сидра будет $L$-отделимым или $L$-регулярным пространством (эти недавно введенные понятия включают аксиомы отделимости $T_{i}$ при $\left.i \in\left\{1,2,2 \frac{1}{3}, 2 \frac{1}{2}, 3,3 \frac{1}{2}\right\}\right)$.
\end{abstract}

1. Introduction. In [1, ex. 9.1], J. Ceder gave an example of a stratifiable space, which is not metrizable. We call this space the Ceder plane and denote it by $\mathbb{M}$. In [2] Ceder's construction was generalized by introducing a general notion of the Ceder product $X \times_{b} Y$ of two topological spaces with a distinguished point $b \in Y$. Important examples of the Ceder product are the Ceder plane $\mathbb{M}=\mathbb{R} \times_{0}[0,+\infty)$, the Alexandroff double circle [3, p. 204] and the Alexandroff duplicate of a given topological space. Topological properties of the Alexandroff duplicate $A D(X)$ of a topological space $X$ have been studied in many papers (see for example [4, 5]). In particular, A. Caserta and S. Watson [4, Corollary 3.7] characterized metrizable subspaces of $A D(X)$.

In [2] it was proved that, if the spaces $X$ and $Y$ are stratifiable then the Ceder product $X \times_{b} Y$ is stratifiable. Some conditions under which the Ceder products are $T_{i}$-spaces for $i \in$ $\{0,1,2,3\}$ were announced in [6] and proved in [7]. Paper [7] also contains a characterization of spaces $X, Y$ with metrizable Ceder product $X \times_{b} Y$.

In this paper we study separation axioms in Ceder products of topological spaces. We accept a general approach of [8] based on the notions of $L$-separated and $L$-regular spaces

2010 Mathematics Subject Classification: 54C08.

Keywords: Separation axioms; linear separation axioms; the Ceder plane; Ceder product; Alexandroff double circle; Alexandroff duplicate; stratifiable space.

doi:10.15330/ms.43.1.78-87

(C) V. K. Maslyuchenko, O. V. Maslyuchenko, O. D. Myronyk, 2015 
for a linearly ordered set $L$. Varying the linearly ordered set $L$ we get the classical separation axioms $T_{i}, i \in\left\{1,2,2 \frac{1}{3}, 2 \frac{1}{2}, 3,3 \frac{1}{2}\right\}$ as partial cases.

2. The Ceder product. Recall that a family $\mathcal{B}$ of open subsets of $X$ is a base for a topological space $(X, \mathcal{T})$ if and only if $\mathcal{B} \subseteq \mathcal{T}$ and for any $G \in \mathcal{T}$ and every point $x \in G$ there exists $B \in \mathcal{B}$ such that $x \in B \subseteq G$. It is known [3, p. 33], that any base $\mathcal{B}$ for $X$ has the following properties:

(B1) for any $B_{1}, B_{2} \in \mathcal{B}$ and every point $x \in B_{1} \cap B_{2}$ there exists $B \in \mathcal{B}$ such that $x \in B \subseteq$ $B_{1} \cap B_{2}$

(B2) for any $x \in X$ there exists $B \in \mathcal{B}$ such that $x \in B$.

Moreover, if a family $\mathcal{B}$ of subsets of $X$ has the properties (B1) and (B2), then the family

$$
\mathcal{T}=\left\{G \in 2^{X}:(\forall x \in G)(\exists B \in \mathcal{B})(x \in B \subseteq G)\right\}
$$

is a topology on $X, \mathcal{B} \subseteq \mathcal{T}$ and $\mathcal{B}$ is a base for the topological space $(X, \mathcal{T})[3, \mathrm{p}$. 46].

For a base $\mathcal{B}$ of the topology of a space $X$ and a point $x \in X$ the family $\mathcal{B}_{x}=\{B \in \mathcal{B}: x \in B\}$ is a neighborhood base of the topology at $x$.

Let $X$ and $Y$ be topological spaces, $F \subseteq X$ and $b \in Y$. For $U \subseteq X$ and $V \subseteq Y$ we put

$$
\dot{V}=V \backslash\{b\}, U \stackrel{F}{\times} V=(U \times V) \backslash(F \times \dot{V}) .
$$

If $F=\{x\}$, then we put $U \stackrel{x}{\times} V=U \stackrel{\{x\}}{\times} V$. Consider a family $\mathcal{B}=\mathcal{B}_{1} \cup \mathcal{B}_{2}$ of subsets of $X \times Y$, where

$$
\mathcal{B}_{1}=\{\{x\} \times \dot{V}: x \in X, V \text { is open in } Y\}
$$

and

$$
\mathcal{B}_{2}=\{U \stackrel{F}{\times} V: U \text { is open in } X, V \text { is open in } Y, b \in V \text { and } F \subseteq X \text { is a finite set }\} .
$$

It is easy to see that the family $\mathcal{B}$ has properties $(B 1)$ and $(B 2)$. Thus the family

$$
\mathcal{T}=\left\{W \in 2^{X \times Y}:(\forall p \in W)(\exists B \in \mathcal{B})(p \in B \subseteq W)\right\}
$$

is a topology on $X \times Y$ and $\mathcal{B}$ is a base for the topological space $(X \times Y, \mathcal{T})$. Note that a neighborhood base at a point $p=(x, y), y \neq b$, consists of all sets $\{x\} \times \dot{V}$, where $V$ is open in $Y$ and $y \in V$; a neighborhood base at a point $p=(x, b)$ consists of all sets $U \stackrel{F}{ } \times V$, where $U$ is an open neighborhood of $x$ in $X$ and $V$ is an open neighborhood of $b$ in $Y$ and $F \subseteq X$ is a finite set such that $x \in F$. Since $U \stackrel{F}{\times} V=U \stackrel{F_{0}}{\times} V$, where $F_{0}=F \cap U$, we can assume that $F \subseteq U$.

The Ceder product of topological spaces $X$ and $Y$ with distinguished point $b \in Y$, denoted by $P=X \times_{b} Y$, is defined to be the topological space $(X \times Y, \mathcal{T})$.

Let $X$ be a topological space and $A D(X)=X \times\{0,1\}$ be the Alexandroff duplicate of $X$ (see $[5,4]$ ). Note that the Alexandroff duplicate $A D(X)$ is the Ceder product $X \times_{0}\{0,1\}_{0}$ of $X$ and the connected doubleton $\{0,1\}_{0}$. The space $\{0,1\}_{0}$ is also called the Sierpiński space.

Put $X_{b}=X \times\{b\}$. Consider the mapping $\varphi: X \rightarrow X_{b}, \varphi(x)=(x, b)$. It is easy to see that $\varphi$ is a homeomorphism of $X$ onto the subspace $X_{b}$ of the Ceder product $P=X \times_{b} Y$. 
For $a \in X$ we put $Y_{a}=\{a\} \times Y$ and $\dot{Y}_{a}=\{a\} \times \dot{Y}$. We consider the mappings $\psi: Y \rightarrow Y_{a}$, $\psi(y)=(a, y)$, and $\psi_{0}=\left.\psi\right|_{\dot{Y}}: \dot{Y} \rightarrow \dot{Y}_{a}$. It is easy to see that $\psi_{0}$ is a homeomorphism of the subspace $\dot{Y}=Y \backslash\{b\}$ of $Y$ onto the subspace $\dot{Y}_{a}$ of the Ceder product $P$. Note, that if the point $b$ is non-isolated in $Y$, then the mapping $\psi$ is not a homeomorphism, because the point $\psi(b)=(a, b)$ is isolated in the subspace $Y_{a}$.

3. $L$-separated and $L$-regular spaces. Let $L$ be a non-empty linearly ordered set, $X$ be a topological space and $x \in X$. An indexed family $\left(U_{\lambda}\right)_{\lambda \in L}$ of open subsets $U_{\lambda}$ of a topological space $X$ is called an $L$-neighborhood of a point $x$ in $X$ if $x \in U_{\lambda}$ for every $\lambda \in L$ and $\bar{U}_{\lambda} \subseteq U_{\mu}$ for any elements $\lambda, \mu \in L$ with $\lambda<\mu$. We say that an $L$-neighborhood $\left(U_{\lambda}\right)_{\lambda \in L}$ of a point $x$ in $X$ separates points $x$ and $u \in X$, if $u \notin U_{\lambda}$ for every $\lambda \in L$. The notation $\left(U_{\lambda}\right)_{\lambda \in L} \prec A$ means that the indexed family $\left(U_{\lambda}\right)_{\lambda \in L}$ is subordinated to a set $A \subseteq X$ i.e. $U_{\lambda} \subseteq A$ for every $\lambda \in L$. It is clear that an $L$-neighborhood $\left(U_{\lambda}\right)_{\lambda \in L}$ of $x \in X$ separates points $x$ and $u \in X$ if and only if $\left(U_{\lambda}\right)_{\lambda \in L} \prec X \backslash\{u\}$.

Following [8], we define a topological space $X$ to be $L$-separated at a point $x \in X$ if for any point $u \in X \backslash\{x\}$ there exists an $L$-neighborhood $\left(U_{\lambda}\right)_{\lambda \in L}$ of $x$ in $X$ such that $\left(U_{\lambda}\right)_{\lambda \in L} \prec X \backslash\{u\}$. Next, a topological space $X$ is said to be $L$-regular at a point $x \in X$ if for any neighborhood $U$ of $x \in X$ there exists an $L$-neighborhood $\left(U_{\lambda}\right)_{\lambda \in L}$ of $x$ in $X$ such that $\left(U_{\lambda}\right)_{\lambda \in L} \prec U$. A topological space $X$ is called $L$-separated (respectively, $L$-regular) if $X$ is $L$-separated (respectively, $L$-regular) at each point $x \in X$.

We add two more properties to the well-known separation axioms $T_{0}, T_{1}, T_{2}, T_{3}, T_{3 \frac{1}{2}}$ (see [3, p. 69]). A topological space $X$ is called a Urysohn space if for any two distinct points $x$ and $y$ of $X$ there are neighborhoods $U$ and $V$ of points $x$ and $y$ respectively, with $\bar{U} \cap \bar{V}=\varnothing$. This property is called the Urysohn separation axiom, which we denoted by $T_{2 \frac{1}{3}}$. A topological space $X$ is called a functionally Hausdorff space if for any two distinct points $x$ and $y$ of $X$ there exists a continuous function $f: X \rightarrow \mathbb{R}$ such that $f(x) \neq f(y)$. We denoted this property by $T_{2 \frac{1}{2}}$.

We will identify each positive integer $n$ with the finite ordinal $\{0,1, \ldots, n-1\}$.

The following result from [8] shows that almost all possible separation axioms are partial cases of $L$-separatedness and $L$-regularity.

Proposition 1. A topological space $X$ is:

(i) a $T_{1}$-space if and only if $X$ is 1-separated;

(ii) Hausdorff (= a $T_{2}$-space) if and only if $X$ is 2-separated;

(iii) Urysohn (= a $T_{2 \frac{1}{3}}$-space) if and only if $X$ is 3-separated;

(iv) functionally Hausdorff ( $=$ a $T_{2 \frac{1}{2}}$-space) if and only if $X$ is $\mathbb{Q}$-separated;

(v) regular if and only if $X$ is 2-regular if and only if $n$-regular for every integer $n \geq 2$;

(vi) completely regular if and only if $X$ is $\mathbb{Q}$-regular;

(vii) Tychonoff (= a $T_{3 \frac{1}{2}}$-space) if and only if $X$ is a $\mathbb{Q}$-regular $T_{1}$-space.

The characterizations in Proposition 1 can be considered as definitions of the corresponding separation axioms.

It is well known [3, p. 114] that any subspace of a $T_{i}$-space is a $T_{i}$-space for $i \leq 3 \frac{1}{2}$. We show that $L$-separatedness and $L$-regularity are hereditary properties too.

Proposition 2. Any subspace $E$ of an $L$-separated space $X$ is $L$-separated. 
Proof. The closure of a set $A$ in $E$ will be denoted by $[A]_{E}$, and the closure of a set $A$ in $X$ will be denoted by $\bar{A}$. It is known [3, p. 111] that $[A]_{E}=\bar{A} \cap E$ for any set $A \subseteq E$.

Let $x$ and $u$ be any distinct points of $E$. Since $X$ is $L$-separated, there exists an $L$-neighborhood $\left(U_{\lambda}\right)_{\lambda \in L}$ of $x$ in $X$ such that $u \notin U_{\lambda}$ for every $\lambda \in L$. We set $V_{\lambda}=U_{\lambda} \cap E$. It is clear that $V_{\lambda}$ is open in $E, x \in V_{\lambda}, u \notin V_{\lambda}$ for every $\lambda \in L$, and $\left[V_{\lambda}\right]_{E}=\bar{U}_{\lambda} \cap E \subseteq U_{\mu} \cap E=V_{\mu}$ for any elements $\lambda<\mu$ of $L$. Therefore $\left(V_{\lambda}\right)_{\lambda \in L}$ is an $L$-neighborhood of $x$ in $E$, which separates points $x$ and $u$. Thus $E$ is an $L$-separated space.

Proposition 3. Any subspace $E$ of an L-regular space $X$ is L-regular.

Proof. Fix $x \in E$ and let $V$ be a neighborhood of $x$ in $E$. There exists a neighborhood $U$ of $x$ in $X$, such that $V=U \cap E$. Since $X$ is an $L$-regular space, there exists an $L$-neighborhood $\left(U_{\lambda}\right)_{\lambda \in L}$ of $x$ in $X$ such that $\left(U_{\lambda}\right)_{\lambda \in L} \prec U$. We put $V_{\lambda}=U_{\lambda} \cap E$ for every $\lambda \in L$. Then $\left(V_{\lambda}\right)_{\lambda \in L}$ is an $L$-neighborhood of $x$ in $E$ such that $\left(V_{\lambda}\right)_{\lambda \in L} \prec V$. Therefore $E$ is $L$-regular.

4. The axioms $T_{0}$ and $T_{1}$ in the Ceder product. We start with finding conditions on spaces $X$ and $Y$ guaranteeing that the Ceder product satisfies the separation axioms $T_{i}$ for $i \in\{0,1\}$.

Theorem 1. Let $X$ and $Y$ be non-empty topological spaces, $b \in Y$ and $P=X \times_{b} Y$ be the Ceder product. Then $P$ is a $T_{0}$-space if and only if $X$ and $\dot{Y}$ are $T_{0}$-spaces.

Proof. Necessity. Since $X$ and $X_{b}$ are homeomorphic, $X$ is a $T_{0}$-space. Similarly, $\dot{Y}$ and $\dot{Y}_{a}$ are homeomorphic, where $a \in X$. So $\dot{Y}$ is a $T_{0}$-space.

Sufficiency. Let $X$ and $\dot{Y}$ be $T_{0}$-spaces. Fix two distinct points $p_{1}=\left(x_{1}, y_{1}\right)$ and $p_{2}=\left(x_{2}, y_{2}\right)$ of $P$. If $x_{1} \neq x_{2}$, then there is an open set $U$ in $X$ containing exactly one of the points $x_{1}, x_{2}$. Then $W=U \times Y$ is open in $P$ and contains exactly one of the points $p_{1}, p_{2}$.

If $x_{1}=x_{2}=x$, then $y_{1} \neq y_{2}$. Assume first that $y_{1}, y_{2} \in \dot{Y}$. Since $\dot{Y}$ is a $T_{0}$-space, there is an open set $V$ in $\dot{Y}$, which contains exactly one of the points $y_{1}, y_{2}$. Thus the set $W=\{x\} \times V$ is a neighborhood of one of the points $p_{1}, p_{2}$ and does not contain the other one.

Finally, consider the case where $y_{1}=b$ or $y_{2}=b$. Suppose, for example, that $y_{1}=b \neq y_{2}$. Then the set $W=X \stackrel{x}{\times} Y$ is a neighborhood of $p_{1}=(x, b)$ which does not contain the point $p_{2}=\left(x, y_{2}\right)$.

Theorem 2. Let $X$ and $Y$ be non-empty topological spaces, $b \in Y$ and $P=X \times_{b} Y$ be the Ceder product. Then $P$ is a $T_{1}$-space if and only if $X$ and $\dot{Y}$ are $T_{1}$-spaces.

Proof. Necessity. Let $P$ be a $T_{1}$-space. As in the proof of Theorem 1 we obtain that $X$ and $\dot{Y}$ are $T_{1}$-spaces.

Sufficiency. Let $X$ and $\dot{Y}$ be $T_{1}$-spaces. Fix two distinct points $p_{1}=\left(x_{1}, y_{1}\right)$ and $p_{2}=\left(x_{2}, y_{2}\right)$ of $P$. If $x_{1} \neq x_{2}$, then there exists a neighborhood $U$ of $x_{1}$, such that $x_{2} \notin U$. Then $W=U \times Y$ is a neighborhood of $p_{1}$ in $P$, and $p_{2} \notin W$.

Consider the case, where $x_{1}=x_{2}=x$. Then $y_{1} \neq y_{2}$. We first consider the subcase $y_{1}, y_{2} \in \dot{Y}$. Since $\dot{Y}$ is a $T_{1}$-space, there exists a neighborhood $V$ of $y_{1}$ in $\dot{Y}$, such that $y_{2} \notin V$. Thus, the set $W=\{x\} \times V$ is a neighborhood of $p_{1}$ in $P$ and $p_{2} \notin W$.

Now let $y_{1}=b \neq y_{2}$. Then the set $W=X \stackrel{x}{\times} Y$ is a neighborhood of $p_{1}=(x, b)$, which does not contain $p_{2}=\left(x, y_{2}\right)$.

Finally, consider the case where $y_{1} \neq b=y_{2}$. The set $W=\{x\} \times \dot{Y}$ is a neighborhood of $p_{1}=\left(x, y_{1}\right)$, which does not contain $p_{2}=(x, b)$. 


\section{L-separatedness of the Ceder product.}

Theorem 3. Let $L$ be a non-empty linearly ordered set, $X$ and $Y$ be non-empty topological spaces, $b \in Y, \dot{Y}=Y \backslash\{b\}$ and $P=X \times_{b} Y$ be the Ceder product. Then the following conditions are equivalent:

(i) $P$ is an $L$-separated space;

(ii) $X$ and $\dot{Y}$ are $L$-separated spaces.

Proof. $(i) \Rightarrow($ ii $)$ Let $P$ be an $L$-separated space. By Proposition 2 , the subspaces $X_{b}=$ $X \times\{b\}$ and $\dot{Y}_{a}=\{a\} \times \dot{Y}$ of the Ceder product $P$ are $L$-separated, where $a \in X$. As in the proof of Theorem 1, we obtain that $X$ and $\dot{Y}$ are $L$-separated.

$(i i) \Rightarrow(i)$ Consider two distinct points $p=(x, y)$ and $q=(u, v)$ of $P$.

Let $x \neq u$. There is an $L$-neighborhood $\left(U_{\lambda}\right)_{\lambda \in L}$ of $x$ in $X$, such that $u \notin U_{\lambda}$ for every $\lambda \in L$, by the $L$-separatedness of $X$. The sets $W_{\lambda}=U_{\lambda} \times Y$ are open neighborhoods of $p$ in $P$ for all $\lambda \in L$, and

$$
\bar{W}_{\lambda} \subseteq \bar{U}_{\lambda} \times Y \subseteq U_{\mu} \times Y=W_{\mu}
$$

for any elements $\lambda<\mu$ of $L$. So, the indexed family $\left(W_{\lambda}\right)_{\lambda \in L}$ is an $L$-neighborhood of $p$ in $P$. Moreover, $q \notin W_{\lambda}$ for every $\lambda \in L$, thus, this $L$-neighborhood separates the points $p$ and $q$.

Now let $x=u$. Then $y \neq v$. Consider the following three logically possible cases.

Let $\{y, v\} \subseteq \dot{Y}$. Since $\dot{Y}$ is an $L$-separated space, there is an $L$-neighborhood $\left(V_{\lambda}\right)_{\lambda \in L}$ of $y$ in $\dot{Y}$, which separates the points $y$ and $v$. The sets $W_{\lambda}=\{x\} \times V_{\lambda}$ are open neighborhoods of $p$ in $P$ for every $\lambda \in L$, and $\bar{W}_{\lambda}=\{x\} \times\left[V_{\lambda}\right]_{\dot{Y}} \subseteq\{x\} \times V_{\mu}=W_{\mu}$ for any elements $\lambda<\mu$ of $L$. Moreover $q \notin W_{\lambda}$ for every $\lambda \in L$. Therefore $\left(W_{\lambda}\right)_{\lambda \in L}$ is an $L$-neighborhood of $p$ in $P$, which separates the points $p$ and $q$.

Let $y=b$. The set $W=X \stackrel{x}{\times} Y$ is clopen in $P, p \in W$ and $q \notin W$. Put $W_{\lambda}=W$ for every $\lambda \in L$. Then the indexed family $\left(W_{\lambda}\right)_{\lambda \in L}$ is an $L$-neighborhood of $p$ in $P$, which separates the points $p$ and $q$.

Finally, consider the case where $v=b$. Then the set $W=\{x\} \times \dot{Y}$ is clopen in $P, p \in W$ and $q \notin W$. Put $W_{\lambda}=W$ for every $\lambda \in L$. The indexed family $\left(W_{\lambda}\right)_{\lambda \in L}$ is the desired $L$-neighborhood.

Therefore the space $P$ is $L$-separated.

From Proposition 1 and Theorem 3 we immediately obtain the following consequence.

Corollary 1. Let $X$ and $Y$ be non-empty topological spaces and $b \in Y$. The Ceder product $P=X \times{ }_{b} Y$ is a Hausdorff (respectively Urysohn, functionally Hausdorff) space if and only if $X$ and $\dot{Y}$ are Hausdorff (respectively Urysohn, functionally Hausdorff) spaces.

6. $L$-regularity of the Ceder product. Recall that a point $x \in X$ of a topological space $X$ is called an accumulation point if every neighborhood $U$ of $x$ in $X$ is infinite.

A point $x \in X$ of a $T_{1}$-space $X$ is accumulating if and only if $x$ is not isolated.

The following theorem provides sufficient conditions of $L$-regularity of the Ceder product.

Theorem 4. Let $L$ be a non-empty linearly ordered set, $X$ and $Y$ be non-empty topological spaces, $b \in Y$ and $P=X \times{ }_{b} Y$ be the Ceder product. Assume that

(i) $X$ and $\dot{Y}$ are $L$-regular spaces and

(ii) if $X$ has an accumulation point, then $Y$ is $L$-regular at the point $b$.

Then the Ceder product $P=X \times_{b} Y$ is $L$-regular. 
Proof. Let $p=(x, y) \in P$ and $W$ be a neighborhood of $p$ in $P$. We are going to find an $L$-neighborhood $\left(W_{\lambda}\right)_{\lambda \in L}$ of $p$ in $P$, such that $\left(W_{\lambda}\right)_{\lambda \in L} \prec W$. First consider the case, where $y \neq b$. There exists a neighborhood $V$ of $y$ in $Y$, such that $\{x\} \times \dot{V} \subseteq W$. The set $\dot{V}=\dot{Y} \cap V$ is a neighborhood of $y$ in $\dot{Y}$. Since $\dot{Y}$ is $L$-regular, there exists an $L$-neighborhood $\left(V_{\lambda}\right)_{\lambda \in L}$ of $y$ in $\dot{Y}$, such that $\left(V_{\lambda}\right)_{\lambda \in L} \prec \dot{V}$. Then the sets $W_{\lambda}=\{x\} \times V_{\lambda}$ are open neighborhoods of $p$ in $P$ for all $\lambda \in L$. Moreover,

$$
\bar{W}_{\lambda}=\{x\} \times\left[V_{\lambda}\right]_{\dot{Y}} \subseteq\{x\} \times V_{\mu}=W_{\mu}
$$

for any elements $\lambda<\mu$ of $L$. Therefore $\left(W_{\lambda}\right)_{\lambda \in L}$ is an $L$-neighborhood of $p$ in $P$. Since

$$
W_{\lambda}=\{x\} \times V_{\lambda} \subseteq\{x\} \times \dot{V} \subseteq\{x\} \times V \subseteq W,
$$

we have $\left(W_{\lambda}\right)_{\lambda \in L} \prec W$.

If $y=b$, then we can find a neighborhood $U \subseteq X$ of $x$, a neighborhood $V \subseteq Y$ of $b$ and a finite subset $F \subseteq U$ such that $U \stackrel{F}{\times} V \subseteq W$.

If $x$ is a non-accumulation point of $X$, then there exists a finite neighborhood $\widetilde{U}$ of $x$ in $X$ such that $\widetilde{U} \subseteq U$. The set $\widetilde{U} \times V=\widetilde{U} \times\{b\}$ is open in $P$. Let $\left(\widetilde{U}_{\lambda}\right)_{\lambda \in L}$ be an $L$-neighborhood of $x$ such that $\left(\widetilde{U}_{\lambda}\right)_{\lambda \in L} \prec \widetilde{U}$. Put $W_{\lambda}=\widetilde{U}_{\lambda} \times\{b\}$ for every $\lambda \in L$. The indexed family $\left(W_{\lambda}\right)_{\lambda \in L}$ is the desired $L$-neighborhood of $p$ in $P$.

Suppose that $X$ is $L$-regular, $x$ is an accumulation point of $X$, then $Y$ is $L$-regular at the point $b$. Then there exist $L$-neighborhoods $\left(U_{\lambda}\right)_{\lambda \in L}$ and $\left(V_{\lambda}\right)_{\lambda \in L}$ of $x$ and $b$ in $X$ and $Y$ respectively, such that $\left(U_{\lambda}\right)_{\lambda \in L} \prec U$ and $\left(V_{\lambda}\right)_{\lambda \in L} \prec V$. The set $W_{\lambda}=U_{\lambda} \stackrel{F}{\times} V_{\lambda}$ is an open neighborhood of $p$ in $P, W_{\lambda} \subseteq W$ for every $\lambda \in L$, and

$$
\bar{W}_{\lambda} \subseteq \bar{U}_{\lambda} \stackrel{F}{\times} \bar{V}_{\lambda} \subseteq U_{\mu} \stackrel{F}{\times} V_{\mu}=W_{\mu}
$$

for any elements $\lambda<\mu$ of $L$. Therefore $\left(W_{\lambda}\right)_{\lambda \in L}$ is an $L$-neighborhood of $p$ in $P$, such that $\left(W_{\lambda}\right)_{\lambda \in L} \prec W$.

A subset $M$ of a linearly ordered set $L$ is called coinitial if for every $\lambda \in L$ there is $\mu \in M$ such that $\mu \leq \lambda$. The smallest cardinality of a coinitial subset in $L$ is called the coinitiality of $L$ and is denoted by $\mathrm{ci}(L)$. A linearly ordered set $L$ is called a set of countable coinitiality if $\operatorname{ci}(L) \leq \aleph_{0}$. It is clear that for a non-empty linearly ordered set $L$ of countable coinitiality we have $\operatorname{ci}(L)=1$ or $\operatorname{ci}(L)=\aleph_{0}$. Indeed, if $M=\left\{\mu_{0}, \mu_{1}, \ldots, \mu_{n}\right\}$ is a finite coinitial subset of a linearly ordered set $L$, where $\mu_{0}<\mu_{1}<\cdots<\mu_{n}$, then the subset $M_{0}=\left\{\mu_{0}\right\}$ is coinitial in $L$. So, in this case we have $\operatorname{ci}(L)=1$. Note that $\mu_{0}$ is the smallest element of the set $L$. If there is no subset of finite coinitiality of a linearly ordered set $L$ and $\operatorname{ci}(L) \leq \aleph_{0}$, then $\operatorname{ci}(L)=\aleph_{0}$. In this case there is a sequence of elements $l_{n}$ in $L$, such that $l_{n+1}<l_{n}$ for every $n \in \mathbb{N}$ and the subset $L_{0}=\left\{l_{n}: n \in \mathbb{N}\right\}$ is coinitial in $L$. For example, $\operatorname{ci}(\mathbb{Q})=\operatorname{ci}(\mathbb{R})=\aleph_{0}$.

In the case where $L$ is an arbitrary linearly ordered set, it is easily seen that if the Ceder product $P=X \times_{b} Y$ is $L$-regular, then $X$ and $\dot{Y}$ are $L$-regular.

For linearly ordered sets $L$ of countable coinitiality the following theorem provides necessary and sufficient conditions of $L$-regularity of the Ceder product.

Let $E \subseteq X \times Y$ and $x \in X$. We denote $E^{x}=\{y \in Y:(x, y) \in E\}$.

Theorem 5. Let $L$ be a non-empty linearly ordered set of countable coinitiality and let $P=X \times_{b} Y$ be the Ceder product of non-empty topological spaces $X$ and $Y$, where $b \in Y$ and $\overline{\{b\}}=\{b\}$. Then $P$ is an $L$-regular space if and only if the following conditions hold: 
(i) $X$ and $\dot{Y}$ are L-regular;

(ii) if $X$ has an accumulation point, then $Y$ is $L$-regular at $b$.

Proof. The "if" part follows from Theorem 4. To prove the "only if" part let $P$ be an $L$-regular space. By Proposition 3, the subspaces $X_{b}=X \times\{b\}$ and $\dot{Y}_{a}=\{a\} \times \dot{Y}$ of the Ceder product $P$ are $L$-regular, where $a \in X$. Since $X$ and $X_{b}$ are homeomorphic, $X$ is $L$-regular. Analogously, $\dot{Y}$ and $\dot{Y}_{a}$ are homeomorphic, so $\dot{Y}$ is $L$-regular.

Assuming that $X$ contains an accumulation point $a \in X$, we shall prove that $Y$ is $L$ regular at $b$. Given any neighborhood $V \subseteq Y$ of $b$, we shall construct an $L$-neighborhood $\left(V_{\lambda}\right)_{\lambda \in L}$ of $b$ in $Y$ with $\left(V_{\lambda}\right)_{\lambda \in L} \prec V$.

If $b$ is isolated in $Y$, then we put $V_{\lambda}=\{b\}$ for every $\lambda \in L$.

We suppose that the point $b$ is non-isolated in $Y$.

If $\operatorname{ci}(L)=1$, then the set $L$ has the smallest element $l \in L$. Since the Ceder product $P$ is $L$-regular, for the neighborhood $W=X \times V$ of the point $c=(a, b)$ in $P$ there is an $L$-neighborhood $\left(W_{\lambda}\right)_{\lambda \in L}$ of $c$ in $P$ such that $\left(W_{\lambda}\right)_{\lambda \in L} \prec W$. For the neighborhood $W_{l}$ of $c$ in $P$ there are neighborhoods $\widetilde{U}$ of $a$ in $X, \widetilde{V}$ of $b$ in $Y$ and a finite subset $F \subseteq U$ such that $\widetilde{U} \times \widetilde{V} \subseteq W_{l}$. Since $a$ is an accumulation point of $X$, there is a point $x \in \widetilde{U} \backslash F$. For every $\lambda \in L$ consider the set $V_{\lambda}=W_{\lambda}^{x}$. We claim that $\left(V_{\lambda}\right)_{\lambda \in L}$ is the desired $L$-neighborhood of $b$ in $Y$.

Since $x \in \widetilde{U} \backslash F$, for any $\lambda \in L$ we have

$$
\widetilde{V}=(\widetilde{U} \stackrel{F}{\times} \widetilde{V})^{x} \subseteq W_{l}^{x} \subseteq W_{\lambda}^{x}=V_{\lambda}
$$

because $l \leq \lambda$. Thus $\widetilde{V} \subseteq V_{\lambda}$, and so $V_{\lambda}$ is a neighborhood of $b$ in $Y$.

For every $\lambda \in L$ we have $W_{\lambda} \subseteq W$. Therefore $V_{\lambda}=W_{\lambda}^{x} \subseteq W^{x}=V$. We prove that $\bar{V}_{\lambda} \subseteq V_{\mu}$ for any elements $\lambda<\mu$ of $L$. We have $\bar{W}_{\lambda} \subseteq W_{\mu}$. We show that $\{x\} \times \bar{V}_{\lambda} \subseteq \bar{W}_{\lambda}$. Given any point $y \in \bar{V}_{\lambda}$, consider the point $p=(x, y)$. Suppose that $y \neq b$. Consider a basic neighborhood $W^{*}=\{x\} \times \dot{V}^{*}$ of $p$ in $P$, where $V^{*}$ is an open neighborhood of $y$ in $Y$. Since the singleton $\{b\}$ is closed in $Y$, the set $\dot{V}^{*}$ is open in $Y$ and $y \in \dot{V}^{*}$, because $y \neq b$. Therefore $\dot{V}^{*}$ is a neighborhood of $y$. Then $\dot{V}^{*} \cap V_{\lambda} \neq \varnothing$. Thus there is $y^{*} \in \dot{V}^{*} \cap V_{\lambda}$. Since $y^{*} \in \dot{V}^{*}$, we have $p^{*}=\left(x, y^{*}\right) \in W^{*}$. On the other hand $y^{*} \in V_{\lambda}=W_{\lambda}^{x}$ and so $p^{*} \in W_{\lambda}$. Therefore $p^{*} \in W^{*} \cap W_{\lambda}$, hence $W^{*} \cap W_{\lambda} \neq \varnothing$. In this way $p \in \bar{W}_{\lambda}$. If $y=b$, then $p=(x, b) \in \widetilde{U} \stackrel{F}{\times} \widetilde{V} \subseteq W_{l} \subseteq W_{\lambda} \subseteq \bar{W}_{\lambda}$.

Consequently,

$$
\bar{V}_{\lambda} \subseteq \bar{W}_{\lambda}^{x} \subseteq W_{\mu}^{x}=V_{\mu}
$$

and so $\bar{V}_{\lambda} \subseteq V_{\mu}$.

Therefore $\left(V_{\lambda}\right)_{\lambda \in L}$ is an $L$-neighborhood of $b$ in $Y$, such that $\left(V_{\lambda}\right)_{\lambda \in L} \prec V$.

Now assume that $\operatorname{ci}(L)=\aleph_{0}$ and fix a strictly decreasing sequence $\left(l_{n}\right)_{n \in \mathbb{N}}$, such that the subset $L_{0}=\left\{l_{n}: n \in \mathbb{N}\right\}$ is coinitial in $L$.

Put $U_{0}=X, V_{0}=V$ and $W_{0}=U_{0} \times V_{0}$. The set $W_{0}$ is a neighborhood of the point $c=(a, b)$ in $P$. By $L$-regularity of $P$, there is an $L$-neighborhood $\left(W_{1, \lambda}\right)_{\lambda \in L}$ of $c$ in $P$, such that $\left(W_{1, \lambda}\right)_{\lambda \in L} \prec W_{0}$. The set $W_{1, l_{1}}$ is a neighborhood of $c$ in $P$. Then there are neighborhoods $U_{1}$ of $a$ in $X, V_{1}$ of $b$ in $Y$ and a finite subset $F_{1} \subseteq U_{1}$ such that $W_{1}=U_{1} \stackrel{F_{1}}{\times} V_{1} \subseteq W_{1, l_{1}}$. The set $W_{1}$ is also a neighborhood of $c$ in $P$. Then there is an $L$-neighborhood $\left(W_{2, \lambda}\right)_{\lambda \in L}$ of $c$ in $P$, such that $\left(W_{2, \lambda}\right)_{\lambda \in L} \prec W_{1}$. Next, we consider the neighborhood $W_{2, l_{2}}$ of $c$ in $P$ 
and choose neighborhoods $U_{2}$ of $a$ in $X, V_{2}$ of $b$ in $Y$ and a finite subset $F_{2} \subseteq U_{2}$ such that $W_{2}=U_{2} \stackrel{F_{2}}{\times} V_{2} \subseteq W_{2, l_{2}}$. For every $n \in \mathbb{N}$ we can construct recursively neighborhoods $U_{n}$ of $a$ in $X, V_{n}$ of $b$ in $Y$, a finite subset $F_{n} \subseteq U_{n}$ and an $L$-neighborhood $\left(W_{n, \lambda}\right)_{\lambda \in L}$ of $c$ in $P$ such that the following conditions are satisfied:

(a) $W_{n+1, \lambda} \subseteq W_{n}=U_{n} \stackrel{F_{n}}{\times} V_{n}$ for every $\lambda \in L$ and $n \in \mathbb{N}$;

(b) $W_{n} \subseteq W_{n, l_{n}}$ for every $n \in \mathbb{N}$.

Note that $U_{n+1} \subseteq U_{n}$ and $V_{n+1} \subseteq V_{n}$ for every $n \in \mathbb{N}_{0}=\mathbb{N} \cup\{0\}$. Indeed, for a fixed $n$ we have

$$
W_{n+1}=U_{n+1} \stackrel{F_{n+1}}{\times} V_{n+1} \subseteq W_{n+1, l_{n+1}} \subseteq W_{n}=U_{n} \stackrel{F_{n}}{\times} V_{n}
$$

Then $U_{n+1} \times\{b\}=W_{n+1} \cap(X \times\{b\}) \subseteq W_{n} \cap(X \times\{b\})=U_{n} \times\{b\}$. It follow that $U_{n+1} \subseteq U_{n}$. Since the point $a$ is an accumulation point of $X$, there is $x \in U_{n+1} \backslash F_{n+1}$. By the above $x \in U_{n}$. Since $W_{n+1} \subseteq W_{n}$ and $x \notin F_{n+1}$, we have $x \notin F_{n}$ because the point $b$ is not isolated. Hence

$$
\{x\} \times V_{n+1}=W_{n+1} \cap(\{x\} \times Y) \subseteq W_{n} \cap(\{x\} \times Y)=\{x\} \times V_{n} .
$$

In this way $V_{n+1} \subseteq V_{n}$. In particular, $V_{n} \subseteq V_{0}=V$ for every $n \in \mathbb{N}$.

Since $U_{n}$ is a neighborhood of the accumulation point $a$ in $X$, we can choose a point $x_{n} \in U_{n} \backslash F_{n}$ for every $n \in \mathbb{N}$. Note that from $W_{n} \subseteq W_{n-1}$ and $x_{n} \notin F_{n}$ it follows that $x_{n} \notin F_{n-1}$ for every $n \in \mathbb{N}$.

For every $\lambda \in L$ there is the smallest number $n=n(\lambda)$ such that $l_{n} \leq \lambda$. For this number $n$ the inequalities $l_{n} \leq \lambda<l_{n-1}$ where $n \geq 2$ and $l_{1} \leq \lambda$ where $n=1$ are true. We put $V(\lambda)=W_{n, \lambda}^{x_{n}}$ for every $\lambda \in L$, where $n=n(\lambda)$. We show that $(V(\lambda))_{\lambda \in L}$ is an $L$-neighborhood of $b$ in $Y$, such that $(V(\lambda))_{\lambda \in L} \prec V$.

Let us verify that $V(\lambda)$ is a neighborhood of $b$ in $Y$. Since $l_{n} \leq \lambda$, we have $W_{n}=$ $U_{n} \stackrel{F_{n}}{\times} V_{n} \subseteq W_{n, l_{n}} \subseteq W_{n, \lambda}$. Then

$$
V_{n}=W_{n}^{x_{n}} \subseteq W_{n, \lambda}^{x_{n}}=V(\lambda)
$$

Therefore, $V_{n} \subseteq V(\lambda)$, and hence the set $V(\lambda)$ is a neighborhood of $b$ in $Y$, because $V_{n}$ is a neighborhood of $b$ in $Y$.

We prove that $V(\lambda) \subseteq V$ for every $\lambda \in L$. Suppose that $n=n(\lambda) \geq 2$ for $\lambda \in L$. Then $l_{n} \leq \lambda<l_{n-1}$ and

$$
W_{n, \lambda} \subseteq W_{n, l_{n-1}} \subseteq W_{n-1}=U_{n-1} \stackrel{F_{n-1}}{\times} V_{n-1}
$$

Since $U_{n} \subseteq U_{n-1}$ and $x_{n} \in U_{n}$, we have $x_{n} \in U_{n-1}$. Note that $x_{n} \notin F_{n-1}$. Therefore

$$
V(\lambda)=W_{n, \lambda}^{x_{n}} \subseteq W_{n-1}^{x_{n}}=V_{n-1} .
$$

But $V_{n-1} \subseteq V$ then $V(\lambda) \subseteq V$. If $n(\lambda)=1$, then

$$
W_{1, \lambda} \subseteq W_{0}=U_{0} \times V_{0} \text { and } V(\lambda)=W_{1, \lambda}^{x_{1}} \subseteq W_{0}^{x_{1}}=V_{0}=V .
$$

Finally, let us prove that $\overline{V(\lambda)} \subseteq V(\mu)$ for any elements $\lambda<\mu$ of $L$. Fix $\lambda \in L$ and verify that $\left\{x_{n}\right\} \times \overline{V(\lambda)} \subseteq \bar{W}_{n, \lambda}$, where $n=n(\lambda)$. Let $p_{n}=\left(x_{n}, y\right)$ be an arbitrary point of $\left\{x_{n}\right\} \times \overline{V(\lambda)}$. We shall prove that $p_{n} \in \bar{W}_{n, \lambda}$. Let $y \neq b$. Consider any neighborhood $V^{*}$ of $y$ in $Y$. The set $W^{*}=\left\{x_{n}\right\} \times \dot{V}^{*}$ is a basic neighborhood of $p_{n}$ in $P$. Note that $\dot{V}^{*}$ is an open 
neighborhood of $y$ in $Y$. Since $y \in \overline{V(\lambda)}$, there is a point $y^{*} \in \dot{V}^{*} \cap V(\lambda)$. It is clear that $p^{*}=\left(x_{n}, y^{*}\right) \in\left\{x_{n}\right\} \times \dot{V}^{*}=W^{*}$ and $p^{*} \in\left\{x_{n}\right\} \times V(\lambda) \subseteq W_{n, \lambda}$. Thus $p^{*} \in W^{*} \cap W_{n, \lambda}$ and hence $p_{n} \in \bar{W}_{n, \lambda}$. If $y=b$, then $p_{n}=\left(x_{n}, b\right) \in U_{n} \stackrel{F_{n}}{\times} V_{n} \subseteq W_{n, l_{n}} \subseteq W_{n, \lambda} \subseteq \bar{W}_{n, \lambda}$.

Let $\lambda, \mu \in L, \lambda<\mu, n=n(\lambda)$ and $m=n(\mu)$. It is clear that $n \geq m$.

Consider the case where $n=m$. Then $V(\lambda)=W_{n, \lambda}^{x_{n}}$ and $V(\mu)=W_{n, \mu}^{x_{n}}$. Since $\bar{W}_{n, \lambda} \subseteq W_{n, \mu}$, we have

$$
\left\{x_{n}\right\} \times \overline{V(\lambda)} \subseteq \bar{W}_{n, \lambda} \subseteq W_{n, \mu} .
$$

Therefore, $\left\{x_{n}\right\} \times \overline{V(\lambda)} \subseteq W_{n, \mu}$, and hence $\overline{V(\lambda)} \subseteq W_{n, \mu}^{x_{n}}=V(\mu)$.

Now let $n>m$. In this case $n>1, n-1 \geq m$ and

$$
\lambda<l_{n-1} \leq l_{m} \leq \mu .
$$

Then $\bar{W}_{n, \lambda} \subseteq W_{n, l_{n-1}} \subseteq W_{n-1} \subseteq W_{n-1, l_{n-1}} \subseteq W_{n-2} \subseteq \cdots \subseteq W_{m} \subseteq W_{m, l_{m}} \subseteq W_{m, \mu}$. Thus, $\bar{W}_{n, \lambda} \subseteq W_{m} \subseteq W_{m, l_{m}} \subseteq W_{m, \mu}$. Therefore

$$
\left\{x_{n}\right\} \times \overline{V(\lambda)} \subseteq \bar{W}_{n, \lambda} \subseteq W_{m},
$$

and we have $\overline{V(\lambda)} \subseteq W_{m}^{x_{n}}=V_{m}$, because $x_{n} \in U_{n} \subseteq U_{m}$ and $x_{n} \notin F_{m}$. Therefore $\overline{V(\lambda)} \subseteq V_{m}$. On the other hand, if $x_{m} \in U_{m}$ and $x_{m} \notin F_{m}$, then

$$
V_{m}=W_{m}^{x_{m}} \subseteq W_{m, \mu}^{x_{m}}=V(\mu),
$$

hence, $\overline{V(\lambda)} \subseteq V_{m} \subseteq V(\mu)$. Thus $\overline{V(\lambda)} \subseteq V(\mu)$.

Corollary 2. Let $X$ be a topological space containing an accumulation point, $Y$ be a topological space, $b \in Y, \overline{\{b\}}=\{b\}$ and $L$ be a non-empty countable linearly ordered set. The Ceder product $P=X \times_{b} Y$ is $L$-regular if and only if $X$ and $Y$ are $L$-regular.

Problem 1. Let $L$ be a non-empty linearly ordered set, $X$ be a topological space containing an accumulation point, $Y$ be a topological space, $b \in Y, \overline{\{b\}}=\{b\}$ and the Ceder product $P=X \times_{b} Y$ be an $L$-regular space. Is it true that $Y$ is $L$-regular at the point $b$ ?

A topological space $X$ is called regular at a point $x \in X$ if for every neighborhood $V$ of $x$ there exists a neighborhood $U$ of $x$ in $X$ such that $\bar{U} \subseteq V$.

A topological space $X$ is called completely regular at a point $x \in X$ if for every neighborhood $U$ of $x$ there exists a continuous function $f: X \rightarrow[0,1]$ such that $f(x)=0$ and $f(u)=1$ for every $u \in X \backslash U$.

From Proposition 1 and Corollary 2 we immediately obtain the following consequence.

Corollary 3. Let $X, Y$ be topological spaces, $X \neq \varnothing, b \in Y$ and $\overline{\{b\}}=\{b\}$. The Ceder product $P=X \times_{b} Y$ is a regular (completely regular) space if and only if the following conditions are valid:

(i) $X$ and $\dot{Y}$ are regular (completely regular) spaces;

(ii) if $X$ has an accumulation point, then $Y$ is regular (completely regular) at $b$.

7. Acknowledgement. The authors are sincerely grateful to Prof. Taras Banakh for his valuable comments that improved the initial version of this paper. 


\section{REFERENCES}

1. Ceder J. Some generalizations of metric spaces, Pacif. J. Math., 11 (1961), 105-126.

2. Maslyuchenko V.K., Myronyk O.D. The Ceder product and stratifiable spaces, Buk. Math. J., 1 (2013), №1-2, 107-112. (in Ukrainian)

3. Engelking R. General Topology, M.: Mir, 1986, 752 p. (in Russian)

4. Caserta A., Watson S. The Alexandroff Duplicate and its subspaces, Applied General Topology, 8 (2007), №2, 187-205.

5. Buzyakova R.Z., Tkachuk V.V., Wilson R.G. A quest for nice kernels of neighbourhood assignments, Comment. Math. Univ. Carolin., 48 (2007), №4, 689-697.

6. Maslyuchenko V., Myronyk O. Stratifiability of the Ceder product, Vseukr. nauk. konf. "Suchasni problemy teorii ymovirnostei ta matematychnogo analizu". Tezy dopovidei, Iv.-Frankivs'k, 2012, 4445. (in Ukrainian)

7. Maslyuchenko V.K., Maslyuchenko O.V., Myronyk O.D. Properties of the Ceder product, Ukr. Mat. Zhurn. (to appear)

8. Banakh T., Ravsky A., Turek S., Kosztolovicz Z. Linear separation axioms, preprint.

${ }^{1}$ Chernivtsi National University

vmaslyuchenko@ukr.net

${ }^{2}$ Chernivtsi National University, Ukraine;

Instytut Matematyki, Akademia Pomorska w Slupsku, Polska

ovmasl@gmail.com

${ }^{3}$ Chernivtsi National University

myronyk.oks@gmail.com 